\title{
Dynamics of proteins and DNA in a Paul trap
}

\author{
Kushal Shah \\ School of Computational and Integrative Sciences, Jawaharlal Nehru University, New Delhi - 110067, India.*
}

\begin{abstract}
Paul traps are increasingly being used in a wide variety of applications in biology including study of protein dynamics and DNA sequencing. In this paper, statistical properties of a macromolecule chain in a Paul trap are theoretically analyzed using a simple model of charged particles connected by rigid rods. It is found that the mean displacement of chain varies linearly with the chain length, showing properties of enhanced diffusion, compared to the normal diffusion relation which exists for the case of a macromolecule chain in a force-free region. The dependence of the mean displacement on the chain length and trap parameters is also found to be very different for the case of pure dc operation and pure ac operation of the Paul trap.

Keywords: peptide chain; end-to-end distance; enhanced diffusion; protein structure
\end{abstract}

\section{INTRODUCTION}

Protein folding and DNA sequencing are two very important areas of research in biology currently. Protein folding has been studied using many different models over the past few decades. Similarly, there are various mechanisms available to sequence a DNA molecule. One common factor that brings these two disparate fields together is the Paul trap [15]. A Paul trap uses a combination of dc and ac fields to trap charged particles and was invented by Wolfgang Paul in 1953. Paul traps have immense applications in mass spectrometry and quantum computation. Over the last two decades, Paul traps have started being extensively used in structural studies of proteins [6-8] and also sequencing of DNA strands [9].

Traditionally, protein structure has been studied using NMR [10] and X-ray crystallography [11]. However, one limitation of NMR is that it can only produce an ensemble averaged image of protein molecules at a given time. Though $\mathrm{X}$-ray crystallography can produce high resolution image of protein molecules, it can only be used to study static structures and not dynamic processes. Using Paul traps, researchers have been able to circumvent both these limitations of NMR and $\mathrm{X}$-ray methods [7]. Apart from this, Paul traps have also been used in the identification of protein sequences [12, 13].

One of the current methods being developed for sequencing of DNA molecules is by using nanopores $[14,15]$. In this technique, it is very important to be able to control the orientation and position of the macromolecule as it passes through the nanopore. It has been recently proposed that a Paul trap can be used to enhance the controllability of a DNA sequence in such a nano-device $[9,16]$. This has the potential to significantly reduce the cost and enhance the speed of DNA sequencing.

In light of the emerging applications of Paul traps in biological problems, it becomes imporant to theoretically analyze the dynamics of a macromolecule chain in this device. A simple model of a protein or DNA sequence is a chain of charged point-particles connected by rigid rods. Though the details of the exact motion of the macromolecule would be interesting to know, an analysis of the statistical properties of the mean

*Electronic address: shah.kushal@yahoo.com displacement of this simple chain model [18] can give useful information from the point of view of applications. Such an analysis has been extensively done previously for the case of a macromolecule chain in a force-free region $[17,19]$. The basic idea used in these previous studies was that of a randomwalk leading to results same as that obtained for Brownian motion [20]. However, as is shown in this paper, the statistical properties of a macromolecule in a Paul trap are very different from that of a macromolecule in a force-free region.

\section{METHODS}

The electrostatic potential in a Paul trap is given by the expression

$$
\Phi(x, y, z, t)=(U+V \cos \omega t)\left(x^{2}+y^{2}-2 z^{2}\right)
$$

where $U, V$ are the parameters governing the dc and ac potentials respectively and $\omega$ is the frequency of the ac potential. Thus, the equations of motion of a free particle in a Paul trap are

$$
\begin{aligned}
& \ddot{x}=\left[-p_{x}+2 q_{x} \cos 2 t\right] x \\
& \ddot{y}=\left[-p_{y}+2 q_{y} \cos 2 t\right] y \\
& \ddot{z}=\left[-p_{z}+2 q_{z} \cos 2 t\right] z
\end{aligned}
$$

where $p_{x}=p_{y}=8 U / \omega^{2}=0.5 p_{z}=p$ (say) and $q_{x}=q_{y}=$ $4 V / \omega^{2}=0.5 q_{z}=q$ (say) (for the sake of simplicity, mass and charge of all particles are taken to be unity). The frequency has been normalized to $\omega=2$ keeping in view the standard Mathieu equation [21].

A simple model for a macromolecule chain is a collection of charged particles held together by rigid rods (see Fig. 1). This model has earlier been used extensively in analysis of protein and DNA dynamics. As shown in Fig. 1, the charged particles of the chain are not completely free to move in any direction. They are contrained by the fact that the distance between two adjacent particles on the chain is a constant.

Consider the macromolecule as a sequence of points, $\left\{\left(x_{i}, y_{i}, z_{i}\right): i=0,1,2, \ldots, N\right\}$. For the sake of simplicity, we consider $\left(x_{0}, y_{0}, z_{0}\right)$ to be fixed at the origin and that the molecule is moving in the $x-z$ plane (so that $y_{i}=0$ for all $i=0,1,2, \ldots, N)$. To solve the equations of motion for the macromolecule, we need to transform to a coordinate system 
without constraints. Let $\eta_{i}=x_{i}-x_{i-1}$ and $\xi_{i}=z_{i}-z_{i-1}$. Thus, the constraint on the system is

$$
r_{i}^{2}=\eta_{i}^{2}+\xi_{i}^{2}=\mathrm{constant}=r^{2} \quad \forall i=1,2,3, \ldots, N
$$

The system can thus be completely defined by a knowledge of $\left\{\theta_{i}\right\}$, which is given by

$$
\tan \theta_{i}=\frac{\xi_{i}}{\eta_{i}}
$$

In cylindrical coordinates, the radial acceleration is given by $a_{r}=\ddot{r}-r \dot{\theta}^{2}$ and angular acceleration by $a_{\theta}=r \ddot{\theta}+2 \dot{r} \dot{\theta}$. In our system, since $r=$ constant, we have $a_{\theta}=r \ddot{\theta}$, which gives

$$
\ddot{\theta}_{i}=\frac{\eta_{i} \ddot{\xi}_{i}-\xi_{i} \ddot{\eta}_{i}}{r_{i}^{2}}
$$

Substituting Eq. (2) in Eq. (5), we get

$$
\begin{aligned}
\ddot{\theta}_{i} & =\frac{1}{2}\left[-p_{z}+p_{x}+2\left(q_{z}-q_{x}\right) \cos 2 t\right] \sin 2 \theta_{i} \\
& =\frac{1}{2}\left[-p_{x}+2 q_{x} \cos 2 t\right] \sin 2 \theta_{i}
\end{aligned}
$$

It should be observed that substituting $p_{y}$ instead of $p_{z}$ in Eq. (6) and using the fact that $p_{y}=p_{x}$ justifies our earlier simplification that the macromolecule chain is moving only in the $x-z$ plane. The charged particles of the polymer chain also interact among themselves electrostatically. However, in this paper, such interactions have been neglected and only the effect of the externally applied electric field of the Paul trap has been considered.

The displacement of the chain can now be given by

$$
d(t)=\sqrt{d_{c}^{2}(t)+d_{s}^{2}(t)}
$$

where

$$
\begin{aligned}
& d_{c}(t)=r \sum_{i=1}^{N} \cos \theta_{i}(t) \\
& d_{s}(t)=r \sum_{i=1}^{N} \sin \theta_{i}(t)
\end{aligned}
$$

A measure of $d$ in Eq. (7) gives a measure of the extent of folding in the chain as a result of its motion inside the Paul trap. For practical purposes, a knowledge of the full timevariation of $d$ is not required. It is enough to know the timeaverage of this displacement. Thus, the quantity that we are interested in is the mean displacement,

$$
\langle d\rangle=\lim _{T \rightarrow \infty} \frac{1}{T} \int_{0}^{T} d(t) \mathrm{d} t
$$

\section{RESULTS}

To analyze the dependence of the mean displacement in Eq. (9) on $p, q, N$, Eqs. (6) and (7) (with $r=1$, without loss of

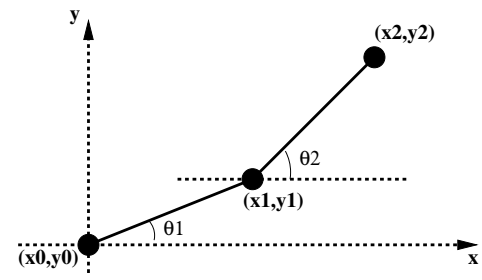

Figure 1: Schematic of a portion of the macromolecule chain.
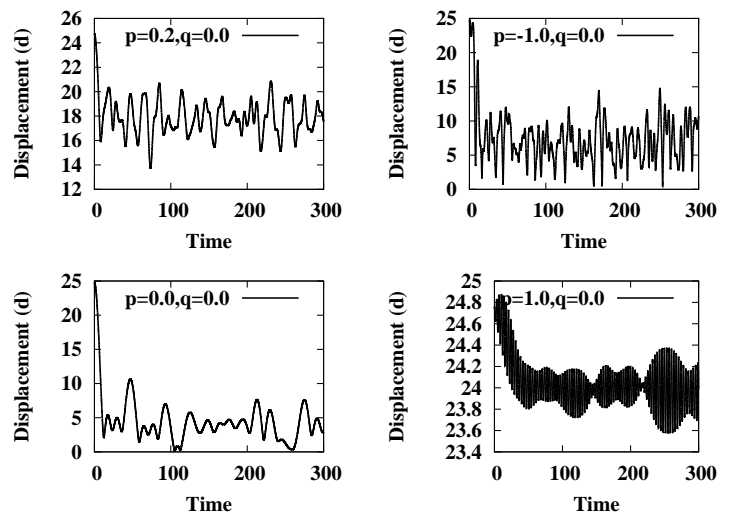

Figure 2: Plot of the displacement given by Eq. (7) with respect to time for various values of $p$.

generality) are solved numerically and the results are now presented. Figure 2 shows the time-variation of $d$ in Eq. (7) for $p=0.0,0.2,1.0,-1.0, q=0.0$ and $N=25$. As can be seen in this figure, the behavior of $d$ is very different for $p>0$ and $p \leq 0$ (when $q=0$ ). For $p>0$, the macromolecule chain displacement tends to be close to its chain length. For $p \leq 0$, the macromolecule chain exists in a folded state with the mean
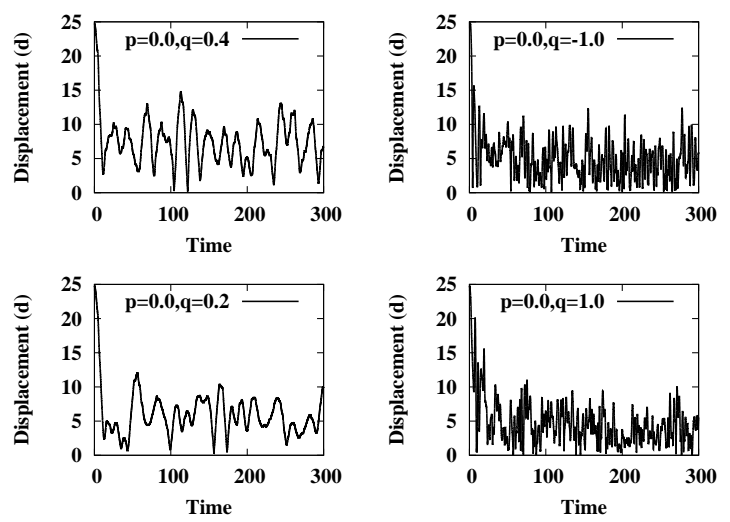

Figure 3: Plot of the displacement given by Eq. (7) with respect to time for various values of $q$. 

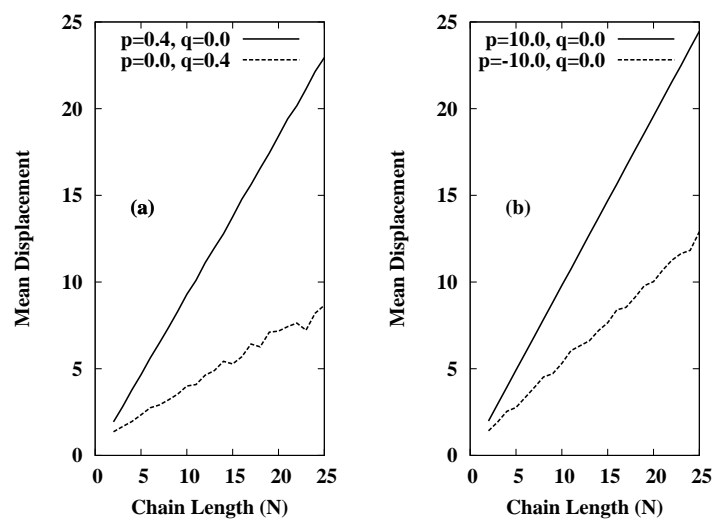

Figure 4: Plot of the ensemble averaged mean displacement in Eq. (9) with respect to chain length for particular values of $p, q$.

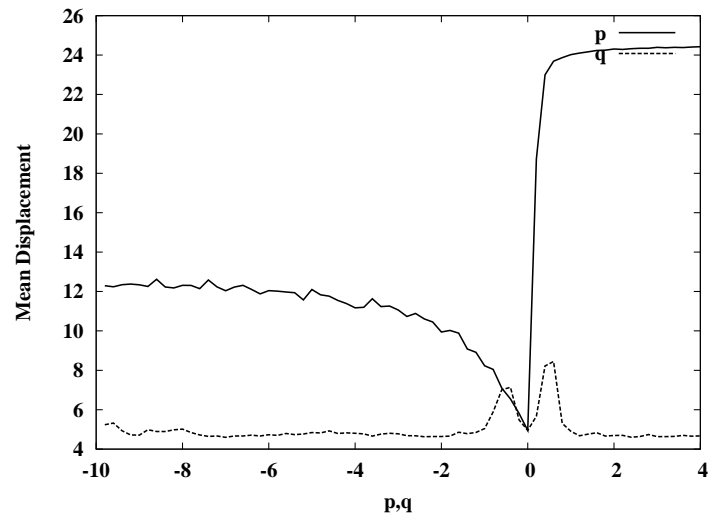

Figure 5: Plot of the ensemble averaged mean displacement in Eq. (9) with respect to $p, q$ for a particular value of $N=25$.

displacement very small compared to the chain length.

Figure 3 shows the variation of $d$ for $p=0.0, q=$ $0.2,0.4,1.0,-1.0$ and $N=25$. As can be seen, the mean displacement of the macromolecule chain is small compared to the chain length for all values of $q$ (when $p=0$ ). Thus, the macromolecule chain exists as a folded structure for all values of $q$. Thus, the behavior of $d$ for $p \leq 0, q=0$ is qualitatively similar to that obtained for various values of $q$ (with $p=0$ ).

Figure 4 shows the mean displacement, $\langle d\rangle$, in Eq. (9) for various values of $N$ and given values of $p, q$. As can be seen, $\langle d\rangle$ varies linearly with the chain length, $N$, enabling us to conjecture

$$
\langle d\rangle \sim N
$$

for all values of $p, q$. Thus, the dependence of $\langle d\rangle$ on $N$ for the case of a Paul trap is similar to that of a system having super-diffusion $[22,23]$. This is very different from that of a macromolecule chain in a force-free region, where the mean displacement scales as square-root of the chain length, $N\left(\langle d\rangle_{\text {free }} \sim N^{0.5}\right.$, which is usual diffusion) [19]. For a macromolecule in a Paul trap, the slope $(\gamma)$ of the linear variation of $\langle d\rangle$ with $N$ will, however, depend on the choice of values for $p, q$. For $p=0, q=0.4$ the slope of the line is $\gamma \approx 0.30$ and for $p=0.4, q=0$, the slope has a much higher value of $\gamma \approx 0.92$.

Figure 5 shows the variation of the mean displacemnt, $\langle d\rangle$, for various values of $p, q$ for a fixed value of $N=25$. As can be clearly seen, the behavior of $\langle d\rangle$ is very different for $p>0$ and $p \leq 0$. However, the dependence of $\langle d\rangle$ is qualitatively similar for positive and negative values of $q$ even though the curve is not exactly symmetric about $q=0$. As shown in Fig. 5, for higher values of $|p|$, the mean displacement saturates to a certain value for a given chain length. This value of $\langle d\rangle_{\text {sat }}$ can be empirically approximated to be equal to the chain length, $N$, for $p \gg 1$ and half of the chain length, $N / 2$ for $p \ll-1$ (see Fig. 4(b)). Thus, there is a clear asymmetry in the behavior of the macromolecule chain for positive and negative values of $p$. However, for larger values of $|q|$, the mean displacement keeps fluctuating about a certain value which is much smaller than the chain length. To average out the deviation and analyze the mean trend in the curve, the plots in Figs. 4 and 5 are shown for an ensemble of 20 macromolecule chains.

\section{DISCUSSION AND CONCLUSION}

In this paper, the statistical properties of a macromolecule chain in a Paul trap have been analyzed and it has been found that the mean displacement, $\langle d\rangle$, of the chain varies linearly with chain length, $N$, which is a sign of super-diffusion. This is very different from the dependence on square-root of chain length (normal diffusion) which is known for a macromolecule in a force-free region.

The dependence of $\langle d\rangle$ on $N$ has been found to be very different for the case of dc operation $(q=0)$ and ac operation $(p=0)$. For the case of ac operation, the macromolecule chain is found to exist in a small folded structure. For the case of dc operation, the chain is found to exist in an unfolded state. This can have important consequences for study of protein structures in Paul traps since protein folding can be assisted or inhibited depending on the values of $p, q$ used in the trap. Also, the protein structure for the case of ac operation needs further analysis since the folded structure of a protein in a Paul trap can be very different from that in solution.

The finding of an unfolded state of the macromolecule chain for dc operation can be very important for DNA sequencing. This is because, in a Paul nano-device, DNA sequences are trapped using pure ac potential. However, when they are to be passed through the nano-pore, dc potential is switched on. And since it is easier to sequence a chain when it exists in its unfolded state, the presence of dc potential can assist in the sequencing of the DNA molecule. 


\section{Acknowledgments}

I would like to thank the Department of Biotechnology of the Government of India for financial support.

[1] W. Paul, Rev. Mod. Phys. 62, 531 (1990)

[2] K. Shah and H. S. Ramachandran, Phys. Plasmas 15, 062303 (2008)

[3] K. Shah and H. S. Ramachandran, Phys. Plasmas 16, 062307 (2009)

[4] H. Schaaf, U. Schmeling, and G. Werth, Appl. Phys. 25, 249 (1981)

[5] M. Sudakov, Int. J. Mass Spectrom. 206, 27 (2001)

[6] E. R. Grant and R. G. Cooks, Science 250, 61 (1990)

[7] M. Sharon and C. V. Robinson, Annu. Rev. Biochem. 76, 167 (2007)

[8] M. Sharon, J. Am. Soc. Mass Spectrom. 21, 487 (2010)

[9] X. Zhao and P. S. Krstic, Nanotechnology 19, 195702 (2008)

[10] K. Wuthrich, NMR of Proteins and Nucleic Acids (WileyInterscience, New York, 1986)

[11] J. Drenth, Principles of Protein X-Ray Crystallography (Springer-Verlag, New York, 1999)

[12] D. Arnott, W. J. Henzel and J. R. Stults, Electrophoresis 19, 968 (1998)

[13] H. Oberacher, W. Parson, P. J. Oefner, B. M. Mayr and C. G.
Huber, J. Am. Soc. Mass Spectrom. 15, 510 (2004)

[14] D. Branton et. al., Nature Biotechnology 26, 1146 (2008)

[15] J. J. Kasianowicz, E. Brandin, D. Branton and D. W. Deamer, Proc. Natl. Acad. Sci. USA 93, 13770 (1996)

[16] S. Joseph, W. Guan, M. A. Reed and P. S Krstic, Nanotechnology 21, 015103 (2010)

[17] P. J. Flory, Statistical Mechanics of Chain Molecules (InterScience, New York, 1969)

[18] Li Tai Fang, Journal of Theoretical Biology 280, 101 (2011)

[19] A. R. Khokhlov and A. Yu Grossberg, Statistical Physics of Macromolecules (American Institute of Physics Press, New York, 1994)

[20] S. Chandrasekhar, Reviews of Modern Physics 15, 1 (1943)

[21] N. W. McLachlan, Theory and Applications of Mathieu Functions (Oxford University Press, Oxford, 1947)

[22] R. Granek and S. Pierrat, Phys. Rev. Lett. 83, 872 (1999)

[23] A. Caspi, R. Granek, and M. Elbaum, Phys. Rev. E 66, 011916 (2002) 